\title{
CLINICAL AND IMMUNOLOGICAL FEATURES OF INFLAMMATORY DISEASES OF THE PELVIS AND GENITAL ORGANS IN HIV-INFECTED WOMEN
}

\section{Olimova N.I., Ikhtiyarova G.A.}

Bukhara State Medical Institute ,Department of Obstetrics and Gynecology No. 1

\begin{abstract}
The aim of the study is to study the cytokine profile in HIV-infected women with acute vaginitis against the background of complex conservative treatment. Materials and methods of research 55 women aged from 25 to 55 years with acute vaginitis were under our supervision. All women were surveyed for the period from 2018 to 2020, of which 35 women with HIV infection, which constitutes the main group, and 20 women without HIV infection, which constitutes the control group. In all female patients, cytokines (to determine inflammatory processes) (IFN $\gamma$, IL-17) were determined in the peripheral blood serum. IFN $\gamma$, IL17 were determined by enzyme-linked immunosorbent assay using test systems "Vector-Best" of the Russian Federation. Results and discussion: the ratio of IFN $\gamma$ / IL-17 (pro-inflammatory / anti-inflammatory cytokines or Th1 / Th2) in HIV-uninfected women was 2.2. In the presence of a pronounced inflammatory process, that is, in HIV-uninfected women, this indicator was 0.96. After carrying out immunocorrective treatment using Immun-5 (The drug is a balanced mixture of natural biologically active substances, the action of which is aimed at activating the body's immune system. The drug increases the body's resistance in inflammatory diseases. An effective remedy for HIV / AIDS, hepatitis and tuberculosis. 1 capsule 2 times a day for 60 days The drug manifests its effects primarily through the effect on the endogenous interferon system and on the expression of cytokines functionally coupled with interferons - IL-2, IL-4, IL-6, IL-10 and IL-17). In the examined HIV-infected women with nonspecific vaginitis, IFN $\gamma$ approached the control values, the content of IL-17, and after treatment did not normalize, remaining 5.5 times higher than in HIV-uninfected women in the control group, the ratio of IFN $\gamma$ / IL-17, in HIV-infected women of the main group, after treatment this indicator decreased to 0.42. Conclusion: The improvement in the clinical condition of HIV-infected women, along with the suppression of the level of the pro-inflammatory cytokine IFN $\gamma$, was accompanied by the disappearance of signs of inflammation of the genitalia, an improvement in the general condition of HIV-infected women.
\end{abstract}

Keywords:

HIV infection, lymphocytes, immune system, cytokines.

Article Received: 18 October 2020, Revised: 3 November 2020, Accepted: 24 December 2020

\section{Introduction}

The problem of infection in women caused by the human immunodeficiency virus (HIV infection) has been studied in obstetrics and gynecology in Uzbekistan since the early 90s. Acute and chronic inflammatory diseases of the genitalia in HIVpositive women, which are an indicator of acquired immunodeficiency syndrome (AIDS), have been identified and described, the symptoms of genital lesions in HIV-infected women with AIDS have been studied. [1,3,5]

The regularity of the spread of HIV infection largely depends on the level of viral load. The viral load in body fluids is an important factor in increasing the likelihood of acquiring HIV infection through sexual contact. At the onset of the disease, in the period from the defeat of the virus to the period when antibodies to HIV begin to be detected, the likelihood of transmission of the virus is extremely high and therefore when a patient who has recently been infected is involved in sexual intercourse or the joint use of intravenous psychoactive substances, there is a great risk of spreading the infection. Equally, in the final period of the development of the disease, when the level of CD4 + lymphocytes falls below 200 cells per $\mu \mathrm{l}$ or AIDS appears, the associated diseases increase the viral load and the likelihood of transmission of the contact virus [4].

Some researchers consider the level of HIV RNA to be the determining factor in the transmission of the virus. This fact served as the reason for a close study of the likelihood of HIV infection during sexual intercourse without the use 
of contraceptives and initiated a dispute about the possibility of "safe" unprotected sex with people who are HIV-seropositive. The Swiss Commission on AIDS (Eidgenossische Kommission fur AIDS-Fragen, EKAF) suggested that the probability of transmission of HIV infection as a result of sexual contact with HIVinfected patients who receive ART and in whom the level of HIV RNA in blood plasma persists for 6 months should be considered negligible below the detection threshold, if they strictly adhere to the order of taking medications, are regularly observed by a doctor and they do not have any symptoms of other sexually transmitted diseases, and allow them to have unprotected sex [3]. According to EKAF, this recommendation will reduce fears of HIV transmission through sexual intercourse and allowing normal sex life for both HIV-positive and non-HIV-infected people.

The problem of termination of pregnancy and medical abortion in patients with HIV infection is urgent. Among HIV-infected women, the vast majority are of fertile age. Medical abortions, carried out at the request of a woman, are mainly carried out in a hospital for young patients with low social status and level of material well-being, in the early stages of HIV infection, who already have children. [7]

In connection with a significant increase in the number of cases of HIV infection, the likelihood of contact between a gynecologist and HIV-infected patients is increasing. In HIV infection, there are often various manifestations of the disease with damage to the genital organs. Gynecologists, as well as other specialists, already have to take an active part in the diagnosis, treatment of HIV-infected persons, preventive work, which, of course, will require knowledge of the peculiarities of the pathology of the genital organs in HIV infection [2,5]

The variety of clinical manifestations of HIV infection is due to the addition of opportunistic infections, among which fungal, bacterial and viral infections are of the greatest importance. The classic manifestation of HIV infection that a gynecologist may face is the development of acute vaginitis. Vaginitis is one of the most common bacterial infections in women with a normal immune system, but the features of the course of these diseases in immunodeficiency are not well understood $[4,11]$.

In women infected with the human immunodeficiency virus, fungal infections in the genital area caused by Candida spp. and Cryptococcus spp. met significantly more often than uninfected. Due to the fact that HIV-infected women usually had a cellular-functional "imbalance" of the immune system, they had diseases caused by aspergillus, penicilli, mucor and other fungi $[1,3,9]$

HIV infection increases the risk of damage to the external genital organs by the human papillomavirus, contributes to the earlier and more frequent development of severe dysplasia and cervical cancer. Moreover, the higher the degree of immunosuppression and the lower the level of T-helper cells (CD4-lymphocytes), the more often highly oncogenic strains of HPV (human papillomaviruses) and intraepithelial diseases of the cervix uteri, up to cancer, are detected. [8] At the same time, there are opposite opinions, asserting that the likelihood of more frequent occurrence of intraepithelial neoplasia and cervical cancer in patients with HIV infection has not been proven. Thus, the world literature has highlighted the negative role of HIV infection in the development of severe HPV-induced diseases of the cervix, such as CIN (cervical intraepithelial neoplasia) and cancer. However, despite a significant number of works presented in foreign literature, today there is no consensus on the features of the pathogenesis, therapy and prevention of intraepithelial lesions in HIVinfected women, as well as clearly developed tactics for the management of HIV-infected women with cervical disease. uterus $[3,4]$.

Meanwhile, our own experience and the few data from clinical studies that are available today suggest that inflammatory diseases of the genitalia, in acute, chronic and recurrent forms, are common in HIV-infected women, although in most cases, the etiology, symptoms and the course 
of inflammatory diseases of the genitalia in HIVinfected women and in women with a normal immune system is the same, however, a protracted, severe or unusual course of these infections, with frequent relapses, or the isolation of atypical bacterial pathogens (including pathogens of opportunistic infections) should alert doctor regarding possible HIV infection [10]. When women turned to a gynecologist, they paid attention to the presence of diseases of the genital area. But also the presence of opportunistic infections, such as: condilematosis of the anal and perianal region, Kaposi's sarcoma, cervical cancer, etc.), and also drew attention to a history of syphilis, gonorrhea and other diseases that are associated with the risk behavior of women. This alarms the gynecologist in order to send the patient for further examination for HIV infection and its complications.

This disease has long attracted the attention of gynecologists, especially since the time when observations and studies appeared, indicating a link between acute vaginitis and HIV infection. [6,7]

The aim of the study is to study the cytokine profile in HIV-infected women with acute vaginitis against the background of complex conservative treatment.

\section{Materials and research methods}

For the period from January 2018 to March 2020 under our supervision there were 55 women aged 25 to 55 years with acute vaginitis, of which 35 women with HIV infection, which is the main group and 20 women without HIV infection, which made up the control group. The research was conducted on the basis of the AIDS Center, on an outpatient basis. The diagnosis of HIV infection was established on the basis of order No. 480 of the Ministry of Health. The level of CD4lymphocytes in peripheral blood in the examined women of the main group averaged 420, VL (viral load) -1500 copies, received ARV therapy according to the $\mathrm{ABC}+3 \mathrm{TC}+\mathrm{EFV}$ regimen. Complaints of patients with headaches, pain and discomfort in the lower abdomen, discharge from the vagina and burning sensation in the genital area.

All women were examined regardless of the presence of complaints, in addition to standard methods of examination (complete blood count, urine, bacteriological and biochemical studies), we conducted a thorough gynecological examination for all HIV-infected women. All examined women were routinely examined and, as a result of the study, according to the indications, were hospitalized for adequate treatment.

Sick women received traditional antibacterial, anti-inflammatory and local therapy in a hospital setting, then immunocorrection was prescribed, for which the domestic drug Immun-5 was used (The drug is a balanced mixture of natural biologically active substances, the action of which is aimed at activating the body's immune system. body resistance in inflammatory diseases.Effective remedy for HIV / AIDS, hepatitis and tuberculosis.Application of 1 capsule 2 times a day for 60 days. The drug manifests its effects primarily through the effect on the system of endogenous interferons and on the expression of functionally associated interferons of cytokines - IL-2, IL-4, IL-6, IL-10 and IL-17). The level of cytokines (IFN $\gamma$, IL-17) in the serum of peripheral blood was studied by the method of enzymelinked immunosorbent assay using test systems "Vector-Best" (Russia).

\section{Results and its discussion}

The results of a study of pro-inflammatory and anti-inflammatory cytokines in the serum of peripheral blood in women with exacerbation of genital inflammation (vaginitis) are presented in Table1 


\begin{tabular}{|l|l|l|}
\hline Indicators & $\begin{array}{l}\text { Control group without HIV } \\
\text { infection }(\mathrm{n}=20)\end{array}$ & $\begin{array}{l}\text { The main group with HIV } \\
\text { infection }(\mathrm{n}=35)\end{array}$ \\
\hline $\mathrm{IFN} \gamma, \mathrm{pg} / \mathrm{ml}$ & $23,70+5,38$ & $\frac{82,80+25,07}{21,93+5,28}$ \\
\hline$, \mathrm{IL}-17, \mathrm{pg} / \mathrm{ml}$ & $\frac{86,08+25,72}{52,04+15,06}$ \\
\hline
\end{tabular}

\section{Note. In the numerator there are data before treatment, in the denominator - after compared with the control group; * $-P<0.05$.}

The analysis of the obtained results revealed the presence of significant differences between the values of the control group and women of the main group. Thus, in healthy women, the level of IFN $\gamma$ was $23.70 \pm 5.38 \mathrm{pg} /$ $\mathrm{ml}$, while in women of the main group this indicator was $82.80 \pm 25.07 \mathrm{pg} / \mathrm{ml}$. Thus, the level of IFN $\gamma$ in women with acute inflammation of the genitalia was increased by 3.5 times, which indicated the severity of the inflammatory process and the presence of purulent discharge from the genital tract.

According to the literature, activated $\mathrm{T}$ lymphocytes and natural killer cells are the source of IFN $\gamma$. Among T-lymphocytes, both cytotoxic CD8 + and CD4 + helper cells are producers of interferon gamma, however, when the latter differentiate into Th1 and Th2, the ability to produce interferon gamma is retained only by Th1 cells. The most important function of IFN $\gamma$ is its participation in mediating the relationship between lymphocytes and macrophages and in the regulation of the ratio of the cellular and humoral components of the immune response. Being the main product of Th1 cells, IFN $\gamma$ reduces the secretory activity of Th2 cells. Thus, IFN $\gamma$ enhances the development of cellular immunity and suppresses the manifestations of humoral immunity. Therefore, IFN $\gamma$ plays an important role in immunoregulation, being a key cytokine of the cellular immune response and an inhibitor of the humoral immune response.

The level of IL-17 in the group of women in the control group was 7.9 times lower than in patients of the main group. It is known that interleukin-10 has been described as a stimulating factor for B-lymphocytes as it causes B-cell proliferation. It is known from the literature that the main producers of IL-17 are T-helpers of the 2nd class. IL-17 is also synthesized by mast cells and B cell lines. IL-17 suppresses the function of macrophages and their secretion of IL-1, TNF and IL-8, while providing an anti-inflammatory effect. Thus, IL-17 is the main product of Th2 cells and stimulates their differentiation. It causes the proliferation and differentiation of $\mathrm{B}$ and $\mathrm{T}$ lymphocytes, affects the development of hematopoietic cells, macrophages, natural killer cells, basophils, being a functional antagonist of cytokines produced by Th1 cells. IL-17 promotes the development of allergic reactions, has a pronounced anti-inflammatory effect.

Comparative analysis showed that the ratio of IFN $\gamma$ / IL-17 (pro-inflammatory / antiinflammatory cytokines or Th1 / Th2) in healthy women was 2.2. In the presence of a pronounced inflammatory process, that is, in women of the main group, this indicator was 0.96. A pronounced imbalance was observed in the state of the main regulatory cytokines, which was expressed by a sharp rise in the level of antiinflammatory cytokines and suppression of proinflammatory cytokines, which are the main regulators of acute inflammatory conditions. Thus, in acute inflammation of the genitalia (vaginitis), a pronounced stimulation of the production of both pro-inflammatory and anti-inflammatory cytokines occurs, which can be regarded as a necessary condition for protection against an infectious agent and the systemic damaging effect of high concentrations of pro-inflammatory cytokines.

After carrying out immunocorrective treatment with the use of Immun-5 in women with 
nonspecific vaginitis, IFN $\gamma$ approached the control values. As for the content of IL-17, after treatment it did not normalize, remaining 5.5 times higher than in women in the control group.

As noted above, the ratio of IFN $\gamma /$ IL-17, in women of the main group, after treatment, this indicator decreased to 0.42 .

\section{Conclusion}

Thus, the improvement in the clinical condition of HIV-infected women, along with the suppression of the level of the pro-inflammatory cytokine IFN $\gamma$, was accompanied by the disappearance of signs of inflammation of the genitalia, an improvement in the general condition of women (on days 9-10 after standard treatment, the condition of sick women improved, headaches, pain and discomfort in the lower abdomen, vaginal discharge and burning sensation in the genital area have decreased).

However, it should be noted that the revealed change in the level of IL-17 and the violation of the ratio of pro- and antiinflammatory cytokines indicated the presence of an immunodeficiency state, which apparently was manifested by the presence of complications against the background of acute vaginitis.

\section{REFERENCES}

[1] Bugubaeva MM, Kadyrova RM Clinical and epidemiological characteristics of children with perinatal contact for HIV infection // Reference book of a general practitioner. 2015. - No. 3. - S. 54-58.

[2] Burkhanov RA, Mamanov MM Phenotypic risk factors for HIV infection and the development of Acquired Immunodeficiency Syndrome // News of Dermatology and Venereology .- (Central Asian Scientific and Practical Journal). - 2019. - No. 3. - S. 39-41.

[3] Varpakhovskaya IM $\begin{array}{r}\text { Acquired } \\ \text { Immunodeficiency }\end{array}$
Syndrome: immunomodulators, vaccines, new methods of treatment // Remedium. - Moscow, 2015. No. 1. - S. 64-68.
[4] Kubanova AA, Akovbyan VA, Toskin IA Modern concepts of the epidemiological process of sexually transmitted infections and HIV infection // Bulletin of Dermatology and Venereology. - 2016. - No. 6. - S. 1-4.

[5] Kulakov V.I., Baranov I.I. HIV: prevention of mother-to-child transmission. - M., 2017. S. 20.

[6] Merkushkina TA, Mirakhmedova NN, The concentration of cytokines IFN-gamma and IL-4 in HIV / AIDS disease in children and adults // Journal of Theoretical and Clinical Medicine. - Tashkent, 2017.- C.163.

[7] Moskaleva EV Dynamics of immune status indicators against the background of antiretroviral therapy correction in children with perinatal HIV infection // Days of immunology in Siberia: mater. vseros. scientific-practical conf., dedicated. 30th anniversary of the GUNII honey. problems of the North of the SB RAMS and the 25th anniversary of the GU Research Institute of Clinical Immunology of the SB RAMS. Krasnoyarsk, 2016 .-- S. 140-141.

[8] Moskaleva EV Changes in interleukin status indicators in children with perinatal HIV infection against the background of immunocorrection // Questions of modern pediatrics. - 2016. - No. 1. - P. 392-393.

[9] Olimova Nasiba Ismatulloyevna Cytokine status in hiv infected women with inflammatory diseases of the genitals// International Engineering Journal For Research and Development Vol.5. 2020

[10] Narzullaev N.U., Ikhtiyarova G.A., Olimova N.I. Clinical and epidemiological characteristics of HIV-infected children, born to HIV-infected mothers. New den meditsiny 1 (29) 2020 January-March.

[11] N. A. Nuraliyev, G. A. Ikhtiyarova, N. I. Olimova, F. Sh. Oripova. Diagnostic Value Determination of Antibodies to Antigensof Micro-Organisms in Women with Inflammatory Diseases of the Pelvic Organs. American Journal of Medicine and Medical Sciences 2020, 10(2): 124-126 
[12] . Nuraliyev N.A., Olimova N.I., Diagnostic value of the determination of antibodies to antigens of microorganisms in women with inflammatory diseases of the pelvic organs. Problems of biology and medicine 2019. 